\title{
Signature and Angular Momentum in 3d-Cranked HFB states
}

\author{
Makito Oi ${ }^{\mathrm{a}}$, Naoki Onishi ${ }^{\mathrm{ad}}$, Naoki Tajima ${ }^{\mathrm{a}}$, Takatoshi Horibata ${ }^{\mathrm{b}}$ 国

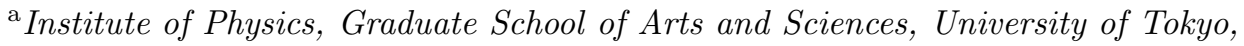 \\ Komaba, Meguro-ku, Tokyo 153, Japan \\ ${ }^{\mathrm{b}}$ Department of Information System Engineering, Aomori University, \\ Kobata, Aomori 030, Japan \\ ${ }^{\mathrm{c}}$ Cyclotron Laboratory, Institute of Physical and Chemical Research (RIKEN), \\ Hirosawa 2-1, Wako-city, Saitama 351-01, Japan
}

\begin{abstract}
In terms of the exact angular momentum projection, properties of the three dimensional cranked HFB (3d-CHFB) states are analyzed quantitatively in the context of the relation between the signature of an intrinsic symmetry and the parity of angular momentum, $(-1)^{I}$. We found that the tilted states have favorable features to describe states involved with high$K$ quantum number and/or odd total angular momentum $I$. This implies that $3 \mathrm{~d}-\mathrm{CHFB}$ can describe properly the backbending phenomena like a "t-band and g-band" crossing, which is suggested in $N=106$ isotones.

Keywords: signature, tilted axis rotation, angular momentum projection
\end{abstract}

In microscopic descriptions of rotational motions of atomic nuclei, the cranked Hartree-FockBogoliubov(CHFB) method has been a useful and practical approach. It has been successful in analyzing the regular rotational spectra [1].

After the discovery of the backbending phenomena, the validity of the method for the application to this angular momentum region came into questions [2, 3]. It is because the standard cranking model is based on a semiclassical picture of a deformed wave packet uniformly rotating about an axis. In such a state, the total angular momentum is highly mixed. This mixing may not be a problem in regular rotational bands, in which the intrinsic state changes gradually with increase of angular momentum. On the other hand, the backbending phenomenon involves an abrupt change of structure taking place in a rather short interval of angular momentum due to a crossing of the ground-state band (g-band) with the rotational aligned band (s-band) 顿. Therefore, the cranking model may not be so adequate in the backbending region as the regular rotational bands. Self-consistent treatments are expected to mend partially this shortcoming.

Hara, Hayashi and Ring [5] applied the method of angular momentum projection to the CHFB states to examine a quality of the states around the backbending region. Their studies are based on the cranking model for rotation about a principal axis of quadrupole deformation (principal axis rotation; PAR). As we will show soon, the PAR-CHFB produces states having mainly even- $I$ and low $K$-quantum number components. This implies that PAR-CHFB is a method analyzing rotational bands associated with even- $I$.

\footnotetext{
${ }^{1}$ e-mail address: mon@nt2.c.u-tokyo.ac.jp

${ }^{2}$ e-mail address: onishi@onishi2.c.u-tokyo.ac.jp

3 e-mail address: tajima@nt1.c.u-tokyo.ac.jp

${ }^{4}$ e-mail address: horibata@aomori-u.ac.jp
} 
With the intention of exploring yrare states which involve high- $K$ and/or odd- $I$ states, a threedimensional self-consistent cranking model was proposed [6], and a schematic calculation along the line was carried out recently on the basis of HFB for ${ }^{182}$ Os [7]. The calculation gives rise to interesting results such as a "tilted axis rotating (TAR)" state as a yrast state. In this paper, we study the nature of 3d-CHFB states, which include both PAR and TAR states, by means of the angular momentum projection. We discuss whether the states are adequate for description of a new type of back bending phenomena caused by "tilted rotation" 8, 9].

Walker et al. [8, 10] claimed from an experimental point of view ( in ${ }^{180} \mathrm{~W}$ and ${ }^{182} \mathrm{Os}$; isotones of $\mathrm{N}=106$ ) that there is a high- $K$ band $\left(K^{\pi}=8^{+}\right)$interacting with the g-band and the s-band one after another at $I \sim 16$ in a quite short interval of angular momentum. The yrast becomes the s-band at high spin and the high- $K$ band comes closer to the yrast. It is possible that the high- $K$ band becomes the yrast before the s-band comes down. They speculate that the high- $K$ band can be a "t-band" because the Fermi energy of such nuclei is located in the middle of high- $j$ $\left(\mathrm{i}_{13 / 2}\right)$ shell and the situation may result in "Fermi alignment" to produce TAR 11]. Such a new type of backbending caused by the "g-t band crossing" may exhibit a characteristic pattern of the signature splitting and, possibly, a signature inversion.

We define in this paper the signature as the symmetry of the $\pi$-rotation about the 1 -axis, which is one of principal axes of the quadrupole moments. For PAR, the rotating axis coincides with the 1-axis; the conventional definition of the signature [1]. For TAR, the rotating axis is tilted by angle $\theta$ towards the 3 -axis.

In our previous work [12], we estimated the signature splitting in terms of the generator coordinate method, in which $\theta$ is chosen as a generator coordinate. We defined the signature as the mirror symmetry with respect to the equatorial plane. In that calculation employing one-dimensional generator coordinate, our definition of the signature is equivalent to the present definition. We assumed that the symmetric(antisymmetric) states are assigned to the even- $I$ (odd- $I$ ) states. This assignment is not obviously accepted and should be examined. In this paper, it is our aim to evaluate quantitatively relationship of signature and the parity of $I$ [13].

We construct the intrinsic state $|\phi\rangle$ based on a variational calculation with a generalized BCS wave function that is a vacuum of the corresponding quasiparticles; $a_{i}|\phi\rangle=0$. The creation and annihilation operators of the quasiparticles $\left(a_{i}^{\dagger}, a_{i}\right)$ are related to those of the nucleons $\left(c_{m}^{\dagger}, c_{m}\right)$ via the generalized Bogoliubov transformation,

$$
\left(\begin{array}{c}
a_{i} \\
a_{i}^{\dagger}
\end{array}\right)=\mathcal{W}^{\dagger}\left(\begin{array}{c}
c_{m} \\
c_{m}^{\dagger}
\end{array}\right), \quad \text { with } \quad \mathcal{W}=\left(\begin{array}{cc}
U_{m i} & V_{m i}^{*} \\
V_{m i} & U_{m i}^{*}
\end{array}\right)
$$

where $\mathcal{W}$ is a unitary matrix. Then, we solve the variational equation with constraints,

$$
\delta\left\langle\phi\left|\left[\hat{H}-\sum_{k=1}^{3}\left(\mu_{k} \hat{J}_{k}+\xi_{k} \hat{B}_{k}\right)-\sum_{\tau=\pi}^{\nu} \lambda_{\tau} \hat{N}_{\tau}\right]\right| \phi\right\rangle=0
$$

where $\hat{J}_{k}$ and $\hat{B}_{k}$ is the $k$ th component of total angular momentum and the "boost" operators [6], 
respectively, and $\hat{N}_{\tau}$ is a nucleon number operator. The boost operators are expressed by mass quadrupole tensors $\hat{Q}_{i j}$ as,

$$
\hat{B}_{k}=\frac{1}{2}\left(\hat{Q}_{i j}+\hat{Q}_{j i}\right) \quad(i j k ; \text { cyclic }),
$$

and the constraint reads, $\left\langle\phi\left|\hat{B}_{k}\right| \phi\right\rangle=0$, acting to fix the principal axes of the quadrupole deformation. In practice, we solve this equation by the method of steepest descent. More detailed procedures to generate wave functions are presented in Ref. 114].

Next, we calculate angular momentum projection matrices.

$$
n_{K K^{\prime}}^{I}\left(\theta, \theta^{\prime}\right)=\left\langle\phi(\theta)\left|\hat{P}_{K K^{\prime}}^{I}\right| \phi\left(\theta^{\prime}\right)\right\rangle \quad \text { with } \quad \hat{P}_{K K^{\prime}}^{I}=\frac{2 I+1}{8 \pi^{2}} \int d \Omega D_{K K^{\prime}}^{* I}(\Omega) \hat{R}(\Omega) .
$$

Here, $\hat{R}(\Omega)$ is a rotation operator through the Euler angles, $\Omega \equiv(\alpha, \beta, \gamma)$, and $D_{K K^{\prime}}^{I}(\Omega)=\langle I K|$ $\hat{R}(\omega)\left|I K^{\prime}\right\rangle$ is the Wigner's function. The states $|\phi(\theta)\rangle$ and $\left|\phi\left(\theta^{\prime}\right)\right\rangle$ are CHFB solutions with tilting angles $\theta$ and $\theta^{\prime}$, respectively. Integration is written as,

$$
\int d \Omega \equiv \int_{0}^{2 \pi} d \alpha \int_{0}^{\pi} \sin \beta d \beta \int_{0}^{2 \pi} d \gamma
$$

The overlap kernels, $\left\langle\phi(\theta)|\hat{R}(\Omega)| \phi\left(\theta^{\prime}\right)\right\rangle$, are evaluated by using the formulae [15],

$$
\left\langle\phi(\theta)|\hat{R}(\Omega)| \phi\left(\theta^{\prime}\right)\right\rangle=\sqrt{\operatorname{det}|P(\Omega)|},
$$

where

$$
P(\Omega)=U(\theta)^{\dagger} D^{\dagger}(\Omega) U\left(\theta^{\prime}\right)+V(\theta)^{\dagger} D^{T}(\Omega) V\left(\theta^{\prime}\right) .
$$

Calculation of the norm kernel has to be carried out with a special caution on choosing the branches of the square root appearing on r.h.s. of eq.(6). In the present work, integration in eq.(4) has to be calculated straightforwardly due to the loss of symmetries, which are related to signature, reality of intrinsic states and conjugation of bra and ket. For details of these symmetries, see Ref. [5].

The intrinsic state and the projection operator can be expanded in terms of a complete orthonormal set of angular momentum,

$$
|\phi\rangle=\sum_{I K \alpha} g_{K \alpha}^{I}|I K \alpha\rangle \quad \text { and } \quad \hat{P}_{K K^{\prime}}^{I}=\sum_{\alpha}|I K \alpha\rangle\left\langle I K^{\prime} \alpha\right|
$$

where $\alpha$ indicates labels additional to $I$ and $K$. The probability $w_{K}^{I}$ found in $|I K\rangle$ is written as,

$$
w_{K}^{I}=\sum_{\alpha}\left|g_{K \alpha}^{I}\right|^{2}=n_{K K}^{I}
$$

and therefore the probability to find states having a certain value of $I$, is evaluated as,

$$
W^{I}=\sum_{K=-I}^{I} w_{K}^{I}=\operatorname{Tr}\left(n^{I}\right) .
$$


Then, we carry out numerical calculations. Let us take a look at the nature of the wave functions generated by 3d-CHFB. Fig.1(a) and Figs.2(a,b) show the probability distributions of $I$ and $K$ in PAR states. The PAR state is obtained under a constraint $\left\langle J_{x}\right\rangle=13 \hbar$, which is in the band crossing region between the g-band and the s-band [14. One observes quite a regular distribution like a Gaussian in the present calculation.

We calculate $\sum_{I} W^{I}$ up to $I=26$; the sum to be 0.96 and 0.86 for $\left\langle\hat{J}_{x}\right\rangle=6$ and 13 , respectively. We take only $36 \times 91 \times 36=117936$ integral points $\left(\Delta \alpha=\Delta \gamma=10^{\circ}, \Delta \beta=2^{\circ}\right)$, so that we can not obtain values of $N_{K K}^{I}$ for very high $I$ and $|K|$ values.

In the PAR for $\left\langle\hat{J}_{x}\right\rangle=J$ and $\left\langle\hat{J}_{z}\right\rangle=\left\langle\hat{J}_{y}\right\rangle=0$, there is a definite signature. Therefore, one would expect that the state contains only even- $I$. As seen in Fig.1(a), this holds quantitatively well. Even- $I$ components occupy $95 \%$ for $\left\langle\hat{J}_{x}\right\rangle=13 \hbar$. However, one can see that the odd components are also included though their fractions are quite small. This is attributed to $\gamma$-deformation associated with the self-consistent field; The wave function for $\langle\boldsymbol{J}\rangle=0$ contains only even- $I$ states because $K=0$ and the $R_{1}$-symmetry. The PAR states are generated by the operator $-\mu_{1} \hat{J}_{1}$, which does not mix states of the different angular momenta. However, our cranking procedure is self-consistent and induces $\gamma$-deformation, which causes mixing of odd- $I$ states. From these facts it is clearly stated that the signature is not an exact but effective quantum number to distinguish the states having even $I$ from odd $I$.

In Figs.2(a,b) presenting the probability distribution $w_{K}^{I}$, the intrinsic states consist mainly of the components with $K=0$, but there is a finite size of fluctuation around $K \sim 0$. This fluctuation can be explained in terms of quantum fluctuation due to the non-commutative nature among the angular momentum components and the induced $\gamma$-deformation. Because the fluctuation is small, the signature seems effective in PAR-CHFB states. In this sense, it is reasonable to employ the PAR cranking model to describe nuclear rotation in the yrast line. However, it is obvious that the PAR intrinsic states are inappropriate for describing the backbending phenomena caused by "g-t band crossing" because of so small amount of odd $I$.

Fig.1(b) and Figs.2(c,d) illustrate the probability distribution of $I$ and $K$ in TAR states, which are obtained under constraints on the total angular momentum; $\left\langle J_{1}\right\rangle=13 \hbar \cos \theta,\left\langle J_{2}\right\rangle=0$ and $\left\langle J_{3}\right\rangle=13 \hbar \sin \theta$. Several characteristic features missing in the PAR states are found . In Fig.1(b) presenting the probability distribution $W^{I}$, one can see that more amounts of odd- $I$ components are contained in the TAR state than in the PAR. Conversely, the amount of the even- $I$ components is decreased to $83 \%$ for $\theta=6^{\circ}$. In Figs.2(c,d) presenting the probability distribution $w_{K}^{I}$ for $\theta=6^{\circ}$, one can see that, although $K \sim 0$ components are still dominant, $K \sim 6$ components are also sizable. For a negative tilting angle, the second peak appears at $K \sim-6$.

These are explained by the fact that the TAR breaks the symmetry of the signature of intrinsic state by tilting the rotating axis. As a consequence, the TAR state contains high- $K$ components and odd- $I$ components as well as even- $I$ low- $K$ components. 
To restore the signature, we try a signature projection of the TAR states,

$$
| \pm\rangle=\mathcal{N}(|+\theta\rangle \pm|-\theta\rangle)
$$

where a normalization factor $\mathcal{N}$ is given by $1 / \sqrt{2(1 \pm \operatorname{Re}\langle\theta \mid-\theta\rangle)}$ and $| \pm\rangle$ express states with $( \pm)$ signature.

After the signature projection, we achieve the angular momentum projection. Fig.1(c) and Figs2.(e-h) show the probability distributions of $I$ and $K$ in signature projected TAR states. For the projected state with $(+)$-signature, both profiles of $W^{I}$ and $w_{K}^{I}$ resemble to those of the PAR state. Even- $I$ components occupy $89 \%$. One can understand this situation as that the tilting angle is small $\left(6^{\circ}\right)$ and the PAR state has $(+)$-signature. A different feature between them is that the projected state with $(+)$-signature contains more amount of high- $K$ components than the PAR. The projected state with (-)-signature exhibits distinguished profiles from any of the other states. Even- $I$ and odd- $I$ components are mixed evenly ( $50 \%$ for the even- $I$ ) and $K \sim 5$ components appear. Incidentally, in Figs.2(g,h), there are tiny peaks at $K=0$. These peaks are artifacts due to numerical errors.

When the overlap matrix $N_{K}^{I} K^{\prime}$ is diagonalized,

$$
\sum_{K^{\prime}} N_{K K^{\prime}}^{I} g_{K^{\prime}}^{I \nu}=n_{\nu}^{I} g_{K}^{I \nu}
$$

the eigenvalues have information on the degree of linear independence of the wave functions, which is called multiplicity 16]. The multiplicity is defined as,

$$
m(I)=\frac{\left(\sum_{\nu} n_{\nu}^{I}\right)^{2}}{\sum_{\nu}\left(n_{\nu}^{I}\right)^{2}}=\frac{\left(\operatorname{Tr}\left[n^{I}\right]\right)^{2}}{\operatorname{Tr}\left[\left(n^{I}\right)^{2}\right]}
$$

In table 1 , the probability $W^{I}$, the multiplicity $m(I)$ and the largest eigenvalues $n_{1}^{I}$ are shown for signature unprojected (designated by $(\theta)$ ), (+)-signature and (-)-signature states. In $W^{I}$, about four times larger odd- $I$ component is included in the $(-)$-signature state than the $(+)$-signature one while about twice larger even- $I$ components is included in the $(+)$-signature state than the (-)-signature one. The multiplicity shows that every state, except for odd- $I(+, \theta)$ states, consists mainly of a state with $n_{1}^{I}$ because values of the $m(I)$ are close to 1 . Indeed, one can see that $W^{I}$ and $n_{1}^{I}$ are close in these cases.

\section{Table 1}

There are two conclusions from the present analysis. First, the signature is an effective quantum number associated with an intrinsic symmetry to distinguish between even and odd $I$ states. However, the resolution is not high enough to identify even-odd $I$ in practical discussion of signature splitting in "g-t band crossing". The angular momentum projection is necessary in the study of yrare states. 
Second, in the backbending region where the high- $K$ band crosses with the g-band, mixing of even-odd $I$ and high- $K$ quantum numbers are highly expected. Considering relative amounts of even and odd total angular momenta, TAR states are much more adequate for treating dynamics involving odd- $I$ states than PAR states.

The numerical calculations are carried out by the Vector Parallel Processor, Fujitsu VPP500/28 at RIKEN. This work is financially supported in part by the Grant-in-Aid for Scientific Research from the Ministry of Education, Science, Sports and Culture of Japan (09640338) 


\section{References}

[1] P. Ring and P. Schuck, "Nuclear Many-Body Problem" (Springer-Verlag 1980)

[2] I. Hamamoto, Nucl. Phys. A271 (1976) 15

[3] F. Grümmer et al., Nucl. Phys. A308 (1978) 77

[4] F.S. Stephens and R.S. Simon, Nucl. Phys. A183 (1972) 257

[5] K. Hara, A. Hayashi and P. Ring, Nucl. Phys. A358 (1982) 14

[6] A. Kerman and N. Onishi, Nucl. Phys. A361 (1981) 179, N. Onishi, Nucl. Phys. A456 (1986) 279

[7] T. Horibata and N. Onishi, Phys. Lett. B325 (1994) 283

[8] P.M. Walker et al., Phys. Lett. B309 (1993) 14

[9] C.J.Pearson et al., Phys. Rev. Lett. 79 (1997) 605

[10] P.M.Walker, private communications

[11] S. Frauendorf, Phys. Scr. 24 (1981) 349

[12] T. Horibata, M. Oi and N. Onishi, Phys. Lett. B355 (1995) 433

[13] N.Tajima, Nucl. Phys. A572 (1994) 365

[14] T. Horibata and N. Onishi, Nucl. Phys. A596 (1996) 251

[15] N. Onishi and T. Horibata, Prog. Theor. Phys 65 (1980) 1650

[16] A. Kerman and N. Onishi, Nucl. Phys. A281 (1977) 373 


\section{Figure Captions}

Fig.1 Probability distributions $W^{I}$. In each graph, even- $I$ and odd- $I$ are drawn separately. (a): PAR states obtained under the angular momentum constraints $\left\langle J_{x}\right\rangle=6 \hbar$ (solid lines), $8 \hbar$ (dash-dot lines), $13 \hbar$ (dash-dot-dot lines) are compared. (b): TAR states with $\theta=0^{\circ}$ (solid lines), $2^{\circ}$ (dash-dot lines) and $6^{\circ}$ (dash-dot-dot lines) are compared. All of the states are obtained under $\left\langle J_{x}\right\rangle=13 \hbar \cos \theta,\left\langle J_{y}\right\rangle=0$ and $\left\langle J_{z}\right\rangle=13 \hbar \sin \theta$. (c): Signature projected TAR states (+/-; solid/dash-dot-dot lines) and a TAR state $(\theta$; dotted line) are compared. Tilting angle is $6^{\circ}$ and the angular momentum constraints are the same as in (b).

Fig.2 Probability distributions $w_{K}^{I}$. (a): even- $I$ graph of a PAR state obtained under $\left\langle J_{x}\right\rangle=13 \hbar$. (b): odd- $I$ graph of the same state as in (a). (c): even- $I$ graph of a TAR state having $\theta=6^{\circ}$. The angular momentum constraints are the same as in Fig.1(b). (d): odd-I graph of the same state as in (c). (e): even- $I$ graph of a $(+)$-signature projected TAR state. The angular momentum constraints and tilting angle are the same as those in Fig.1(c). (f): odd- $I$ graph of the same state as in (e). (g): even- $I$ graph of a (-)-signature projected TAR state. The angular momentum constraints and tilting angle are the same as those in Fig.1(c). (h): odd- $I$ graph of the same state as in $(\mathrm{g})$. 


\section{Table caption}

Table 1 Probability $W^{I}$, multiplicity $m(I)$ and the largest eigenvalue of eq.(12) $n_{1}^{\nu}$ are presented for each state; $(\theta)$ : a TAR state, $( \pm)$ : $( \pm)$-signature projected states. Tilting angle is $6^{\circ}$, and the angular momentum constraint is; $|\langle\boldsymbol{J}\rangle|=13 \hbar$. 
Numerical values of probability, multiplicity and the largest eigenvalue

\begin{tabular}{|l|ccc||l|ccc|}
\hline$I$ ( sign) & $W^{I}$ & $m(I)$ & $n_{1}^{I}$ & $I$ ( sign) & $W^{I}$ & $m(I)$ & $n_{1}^{I}$ \\
\hline $11(\theta)$ & 0.0153 & 1.93 & 0.011 & $12(\theta)$ & 0.0888 & 1.40 & 0.074 \\
$11(+)$ & 0.0108 & 2.64 & 0.006 & $12(+)$ & 0.0960 & 1.22 & 0.087 \\
$11(-)$ & 0.0418 & 1.30 & 0.036 & $12(-)$ & 0.0470 & 1.29 & 0.041 \\
$13(\theta)$ & 0.0181 & 1.71 & 0.014 & $14(\theta)$ & 0.0889 & 1.46 & 0.072 \\
$13(+)$ & 0.0125 & 2.38 & 0.008 & $14(+)$ & 0.0945 & 1.25 & 0.084 \\
$13(-)$ & 0.0511 & 1.24 & 0.046 & $14(-)$ & 0.0555 & 1.26 & 0.049 \\
\hline
\end{tabular}



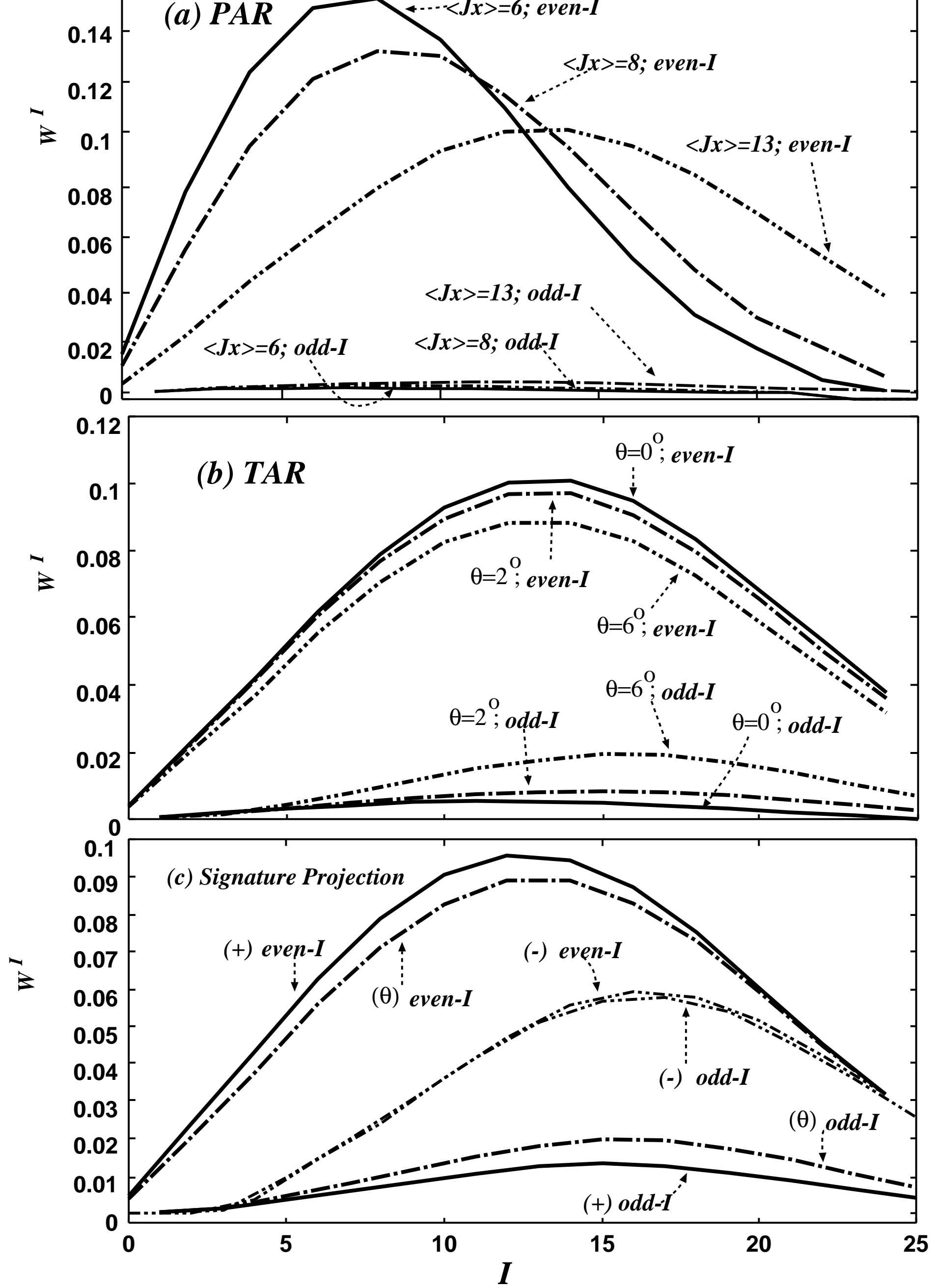
(a)

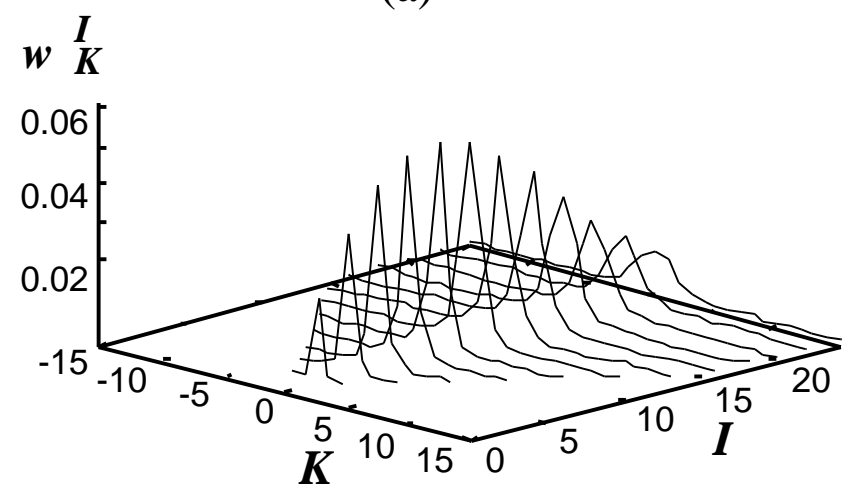

PAR

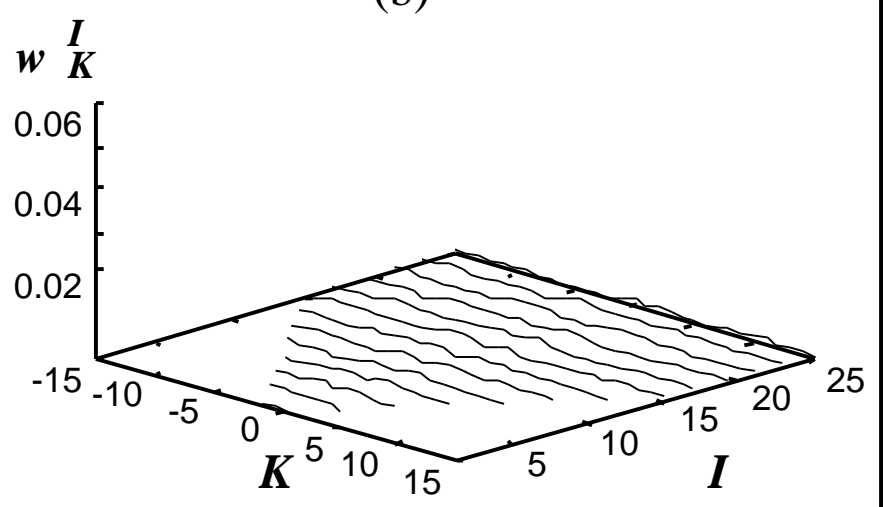

\section{$T A R ;+\theta$}

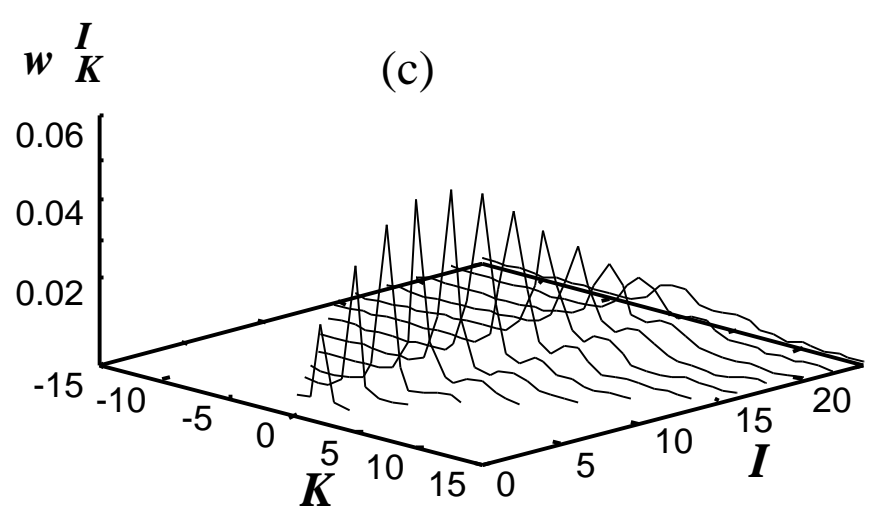

${ }^{w}{ }_{K}^{I}$

(d)
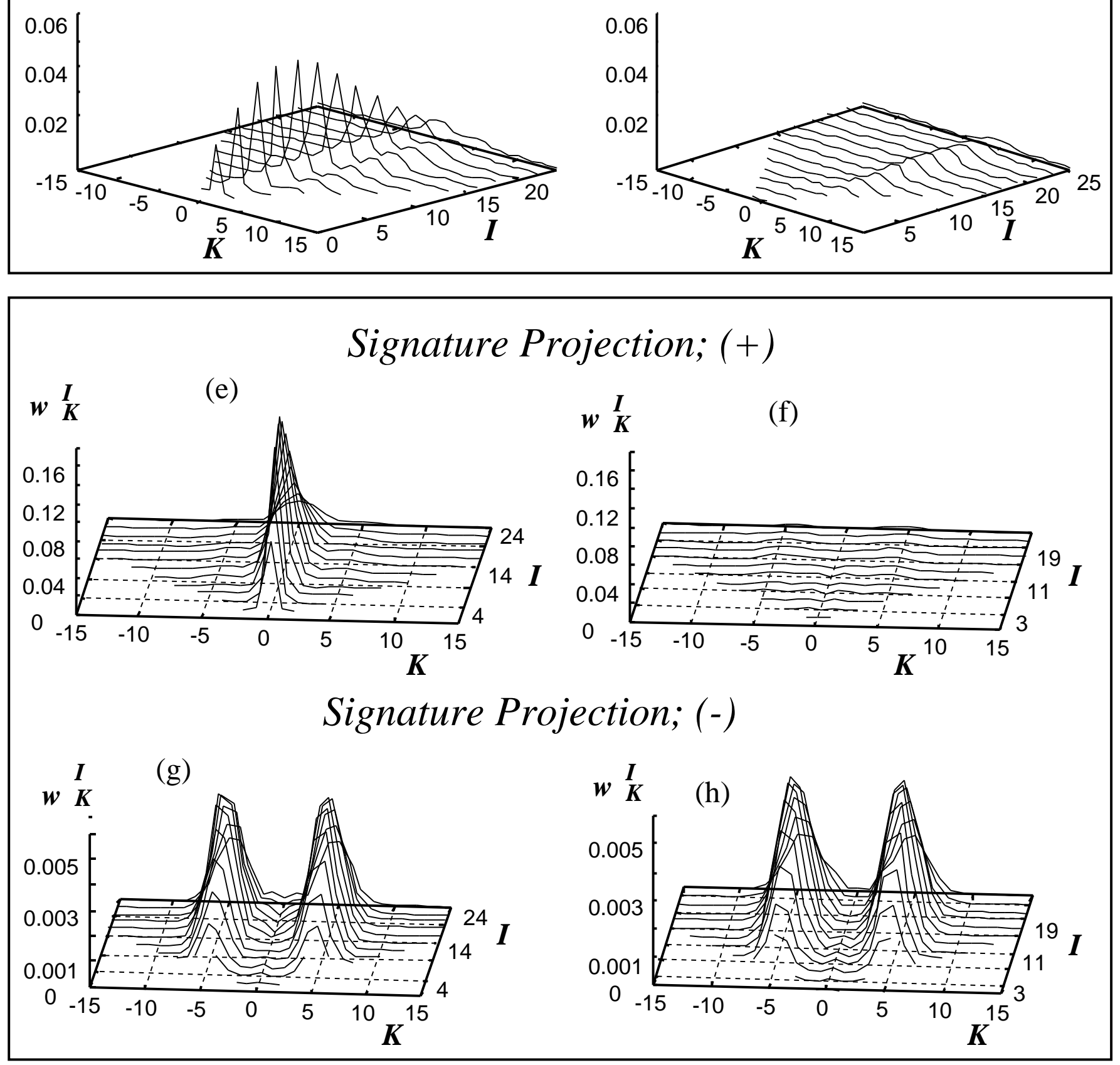